Drug Alcohol Depend. 2015 March 1; 148: 180-187. doi:10.1016/j.drugalcdep.2015.01.002.

\title{
Attention Deficit Hyperactivity Disorder Symptoms and Smoking Trajectories: Race and Gender Differences
}

\author{
Chien-Ti Lee, \\ School of Family Life, Brigham Young University, School of Family Life, JSFB 2083, Provo, UT \\ 84602, United States \\ Trenette T. Clark, \\ School of Social Work, University of North Carolina at Chapel Hill, 325 Pittsboro St., CB \#3550, \\ Chapel Hill, NC 27599, United States \\ Scott Kollins, \\ Department of Psychiatry and Behavioral Sciences, Duke University School of Medicine, 2608 \\ Erwin Road, Suite 300, Durham, NC 27705, United States \\ F. Joseph McClernon, and \\ Department of Psychiatry and Behavioral Sciences, Duke University School of Medicine, 2608 \\ Erwin Road, Suite 300 Durham, NC 27705, United States

\section{Bernard F. Fuemmeler} \\ Department of Community and Family Medicine, Duke University School of Medicine, DUMC \\ 104006, Durham, NC, 27710, United States
}

\begin{abstract}
Purpose-This study examined the influence of Attention Deficit Hyperactivity Disorder (ADHD) symptoms severity and directionality (hyperactive-impulsive symptoms relative to inattentive symptoms) on trajectories of the probability of current (past month) smoking and the number of cigarettes smoked from age 13 to 32 . Racial and gender differences in the relationship of ADHD symptoms and smoking trajectories were also assessed.
\end{abstract}

Methods-A subsample of 9,719 youth ( $54.5 \%$ female) was drawn from the National Longitudinal Study of Adolescent to Adult Health (Add Health). Cohort sequential design and

\footnotetext{
(C) 2015 Elsevier Ltd. All rights reserved.

Correspondence concerning this article should be addressed to Chien-Ti Lee, 2083 JFSB, School of Family Life, Brigham Young University, Provo UT 84602. Phone: (801) 422-4405. Fax: (801) 422-0230. chienti_lee@ byu.edu.

Publisher's Disclaimer: This is a PDF file of an unedited manuscript that has been accepted for publication. As a service to our customers we are providing this early version of the manuscript. The manuscript will undergo copyediting, typesetting, and review of the resulting proof before it is published in its final citable form. Please note that during the production process errors may be discovered which could affect the content, and all legal disclaimers that apply to the journal pertain.

Contributors: Chien-Ti Lee and Bernard Fuemmeler designed the study in conjunct with Joseph McClernon and Scott Kollins. Chien-Ti Lee managed the literature search, undertook the statistical analysis, and completed the first draft of the manuscript. Trenette Clark summarized previous related work and edited the subsequent revisions of the manuscripts. All authors contributed to the editing the final draft of the manuscript and have approved the final version.

Conflict of Interests: None declared.
} 
zero-inflated Poission (ZIP) latent growth modeling were used to estimate the relationship between ADHD directionality and severity on smoking development.

Results-ADHD severity's effect on the likelihood of ever smoking cigarettes at the intercept (age 13) had a greater impact on White males than other groups. ADHD severity also had a stronger influence on the initial number of cigarettes smoked at age 13 among Hispanic participants. The relationships between ADHD directionality (hyperactive-impulsive symptoms relative to inattentive symptoms) and a higher number of cigarettes smoked at the intercept were stronger among Hispanic males than others. Gender differences manifested only among Whites.

Conclusion-ADHD severity and directionality had unique effects on smoking trajectories. Our results also highlight that the risk of ADHD symptoms may differ by race and gender.

\section{Keywords}

Smoking trajectories; ADHD symptoms; racial and gender differences

\section{INTRODUCTION}

Individuals diagnosed with Attention Deficit Hyperactivity Disorder (ADHD) smoke more cigarettes than those without ADHD (Gudjonsson et al., 2012). ADHD symptoms at levels below the threshold for clinical diagnosis adversely influence smoking outcomes (Chang et al., 2012; Elkins et al., 2007; Kollins et al., 2005; Tercyak et al., 2002). The relative contributions of the different ADHD symptom domains (Hyperactive-Impulsive [HI] vs. Inattention [IN]) have been of interest in several studies (Fuemmeler et al., 2007; Kollins et al., 2005; McClernon et al., 2008; Polanczyk et al., 2010; Ranby et al., 2012; Van et al., 2012; Willoughby et al., 2009). Some studies showed IN symptoms are more predictive of smoking outcomes (e.g., Tercyak et al., 2002; Aytaclar et al. 1999; Burke et al, 2001; Molina and Pelham, 2003) than HI; whereas others report HI symptoms are the stronger predictor (e.g., Kollins et al., 2005). Drawing comparisons across studies is difficult given the differences in methods used to assess ADHD symptoms, age of participants, and the smoking behavior. Continued work is needed to better understand the relative role of $\mathrm{HI}$ and IN symptoms on smoking behavior over the course of development.

Race and gender are highly relevant to smoking behavior. Previous studies show that Whites are more likely to become regular or heavy smokers than Blacks (White et al., 2004), whereas Blacks are more likely to continue smoking into adulthood (Kandel et al., 2011). While most studies of smoking focus on Whites and Blacks, Chen and Jacobson (2012) examined the developmental pattern of cigarette smoking among Hispanic, White, and Black adolescents. They documented that Hispanic youth experienced higher rates of smoking during early adolescence compared to White and Black youth, White youth reported increased cigarette use during adolescence, and Black youth showed higher levels of smoking than White youth after entering adulthood. With regard to gender, males smoke significantly more than females during adolescence and adulthood though some exceptions have been noted (Kivinieme et al., 2011; Substance Abuse and Mental Health Services Administration, 2012). For example, Chen and Jacobson (2012) showed that females smoked more than males in early adolescence, but males smoked more than females from 
middle adolescence to adulthood, suggesting that the relation between gender and smoking varies across development. Despite documented differences in smoking behavior by race and gender, little is known about whether gender and race modify the association between ADHD and smoking outcomes.

Utilizing Add Health data, this study extended previous work that only either (a) looked at the relationships between ADHD symptoms and smoking outcomes with gender and ethnicity being controlled (e.g, Fuemmeler et al., 2007), or (b) focused primary on the impact of gender and ethnicity on smoking trajectories (e.g., Chen and Jacobson, 2012). The current study differs from previous studies by investigating the unique contribution of $\mathrm{HI}$ and IN symptoms on smoking trajectories from early adolescence to adulthood as a function of race and gender. A clearer understanding of how race and gender influence the relationship between ADHD symptoms and smoking over development has the potential to elucidate the etiology of smoking, and inform treatment and prevention approaches. This study: (a) examined the overall severity and directionality (HI versus IN) of ADHD symptoms in predicting smoking from ages 13 to 32, and (b) assessed whether race and gender differentially influenced the association between ADHD symptoms and smoking.

\section{METHODS}

\subsection{Participants}

Data were drawn from 14,779 Add Health participants (53.2\% female) interviewed from Waves I to IV. Respondents completed in-home surveys during the 1994-1995 school year (Wave I) and three additional times: Wave II during 1996, Wave III during 2001-2002, and Wave IV during 2008-2009. The participants' mean ages for each of the four waves were $15.65(S D=1.75), 16.22(S D=1.64), 22.96(S D=1.77)$, and $28.9(S D=1.76)$, respectively. Study design and data collection have been described elsewhere (see Harris et al., 2009; Resnick et al., 1997). Due to missing data on the covariates and indicators of ADHD symptoms, and restricting the sample to Whites, Hispanics, and Blacks by excluding small racial groups including "Asians" $(n=936)$, "Native Americans" $(n=263)$, and "Other" $(n=$ 130 ), our analysis sample was further reduced to 9,719 participants.

\subsection{Measures}

2.2.1 Smoking Status-At all waves, participants reported their smoking status using a computer-aided survey instrument. Participants who had never smoked or who had not smoked during the prior 30 days were coded as having smoked 0 cigarettes in the past 30 days (non-current users). The smoking status of current users was coded by their responses regarding to the number of cigarette smoked per day on the days they smoked in the past 30 days.

2.2.2 ADHD Symptoms-At Wave III, participants retrospectively reported on the DSMIV ADHD symptoms that they experienced between ages 5 and 12. Responses were on a 4point scale: (1) "never or rarely," (2) "sometimes," (3) "often," or (4) "very often." A symptom was considered present (coded as 1 ) if it was experienced "often" or "very often," whereas a symptom was considered non-present (coded as 0 ) if it was experienced "never or 
rarely" or "sometimes." One DSM-IV HI ADHD symptom, "Often interrupts or intrudes on others," was not available in the dataset. Accordingly, eight HI symptoms and nine IN symptoms were available for analysis and resulted in uneven number of items for the $\mathrm{HI}$ and IN domains. Therefore, HI and IN latent factor scores were created, representing a continuous score of the levels of symptoms endorsed.

2.2.3 Gender and Race-Male was coded as " 1 " and female as " 2 ". Based on participants' response of their race, mutual exclusive groups were created for White, Hispanic, and Black.

2.2.4 Covariates-Since educational level and conduct problems have been shown to be associated with both ADHD and smoking outcomes (Armstrong and Costello, 2002; Eiraldi et al., 1997), these variables were included as covariates. Parents'/caregivers' education level was coded categorically as less than high school, high school or equivalent, some college, or college degree or beyond. Conduct Problems (CP) was assessed at Wave I by asking participants whether they have engaged in 13 conduct-related problem behaviors (e.g., fighting, property damage, lying to parents or guardians). Depending on the question, responses ranged from 0 (never) to 3 (5 or more times). The 13 items were dichotomized into 0 (never) and 1 (one or more); the sum score served as the $\mathrm{CP}$ measure. A reliability coefficient (KR20) for CP symptoms was 0.71 , suggesting the $\mathrm{CP}$ measure in this study is adequate and the measure has been used in previously published studies with the Add Health database (e.g., Fuemmeler et al., 2013; Kollins et al., 2005; McClernon et al., 2008; Miles et al., 2002).

\subsection{Analytic Procedure}

Analyses were conducted using Mplus version 7.11 (Muthén and Muthén, 1998-2012). We incorporated survey design effects and survey weights into the analysis. We also employed a cohort-sequential design in which age, rather than wave, equaled the unit of time (see Bollen and Curran, 2006; Duncan et al., 2006, 2007). The cohort-sequential approach results in substantial missing data by design; Mplus employs an expectation maximization (EM) algorithm to limit potential biases in such designs (Duncan et al., 2006).

To model smoking, we used latent growth modeling (LGM) along with zero-inflated Poisson (ZIP). LGM-ZIP allows for estimating growth components including the average level of a parameter at starting point (i.e., intercept), the increase (or decrease) rate over time (i.e., linear trend), and deceleration (or acceleration) in the rate of the linear trend (i.e., quadratic trend) for binary (probability of current smoking) and count (number of cigarettes smoked) trajectories. These growth components were further regressed on covariates to determine the association that a particular covariate has with each growth component.

Because HI and IN symptoms are highly correlated, putting these domains in the same model may distort the interpretation of the results. We adopted a procedure proposed by Essex et al. (2006) for dealing with highly correlated domains. Specifically, this procedure bifurcates what the correlated factors have in common [severity $=(\mathrm{HI}+\mathrm{IN}) / 2]$, from what distinguishes them [directionality $=(\mathrm{HI}-\mathrm{IN}) / 2]$. Accordingly, the effect of severity on smoking would be the non-specific effect of ADHD symptoms. The effect of directionality 
would be the specific effect of one domain relative to the other, in this case, HI symptoms relative to IN symptoms.

Subsequent to fitting unadjusted growth curves, the estimated smoking trajectories (i.e., probability of current smoking and the number of cigarettes smoked) were regressed on ADHD severity and directionality, along with gender and racial groups. A multigroup approach was applied to estimate the relationship between ADHD symptoms and smoking trajectories, stratified by racial and gender groups. Invariance of the effects of ADHD symptoms on smoking trajectories across gender and race were tested using a series of Wald's chi-square test to examine whether significant differences exist (a) among racial groups within the same gender or (b) between genders within the same racial groups.

\section{RESULTS}

\subsection{Sample Characteristics}

Around half of the participants were female (54.5\%). The racial groups included Whites (60.9\%), Blacks (22.5\%), and Hispanics (16.5\%). Approximately 24\% of the participants' primary caregivers had earned college degrees. The percentage of participants reporting six or more HI symptoms (2.9\%), six or more IN symptoms (2.5\%), or six or more of both HI and IN symptoms (2.2\%) are similar with previous research (e.g., Tercyak et al., 2002). Figures 1 and 2 show the estimated smoking trajectories over development by race and gender groups.

\subsection{ADHD Symptoms and Smoking Trajectories}

Table 1 shows the results of smoking trajectories (i.e., use vs. non-use and number of cigarettes smoked) regressed on ADHD scores (i.e., severity and directionality) after controlling for gender, race, conduct problems, and primary caregiver's educational level. The adjusted slope for the binary trajectory was $\beta=4.41(S E=.48)$ suggesting that the probability of current smoking increased with age. The negative value for the quadratic ( $\beta=$ $-3.84, S E=.64)$ indicates that the increase in the change in smoking probability decelerated approaching adulthood.

ADHD severity was marginally related to the intercept $(\beta=0.11, p=0.05)$ and slope $(\beta=$ $0.25, p=0.05$ ) of the binary smoking trajectory. This suggests that a higher level of symptom severity predicted the probability of current smoking at intercept and an increasing prevalence of current smoking with age. Similarly, ADHD directionality was significantly related to the slope $(\beta=0.34, p=0.01)$ and quadratic parameters $(\beta=-0.38, p=0.01)$ for the binary trajectory. The positive values for the slope and negative quadratic parameters indicate that increasing HI symptoms relative to IN symptoms increases the probability of current smoking with age with deceleration in this increasing probability over age.

In predicting the count trajectory or the number of cigarettes smoked per day among those who indicated past 30-day smoking, ADHD severity was positively related to the slope ( $\beta=$ $0.60, p=0.01)$ and negatively related to the quadratic terms $(\beta=-0.72, p<0.001)$. This suggests that an increase in ADHD symptoms affect a more rapid increase in the number of 
cigarettes smoked during adolescence, but that this increase decelerates rapidly as individuals approach adulthood (i.e., returns to baseline).

\subsection{ADHD Symptoms and Smoking Trajectories Stratified by Gender and Race}

Table 2 presents the results regarding the influences of ADHD severity and directionality on smoking trajectories, stratified by gender and race. The results show different patterns by which ADHD severity and directionality relate to the binary trajectory (estimated prevalence of current smoking vs non-smoking) and count trajectory (estimated number of cigarettes smoked on the day participants smoked) with respect to gender and race.

3.3.1 Binary trajectory—For White males, White females, and Hispanic females, ADHD severity was related to the binary growth parameters. ADHD severity was significantly related to a higher likelihood of current smoking at the intercept among White males ( $\beta=$ $0.32, p=0.005$ ), suggesting that ADHD severity is a much more salient predictor of probability of current smoking for White males at age 13. Although ADHD severity was not related to the intercept for White and Hispanic females, it was related to the slope for both groups ( $\beta=0.50$ for White females; $\beta=0.60$ for Hispanic females) indicating that severity of ADHD symptoms remains important in predicting the increase in the probability of current smoking over developmental age.

Except for White males and Hispanic females, ADHD directionality was related to the slope, indicating HI symptoms relative to IN symptoms, were related to the increase in the probability of current smoking over age ( $\beta=0.63$ for Hispanic males; $\beta=0.70$ for Black males, $\beta=0.39$ for White females; $\beta=0.58$ for Black females).

3.3.2 Count trajectory-ADHD severity predicted the count trajectory (number of cigarettes smoked per day among those who smoked in the last 30 days) similarly in all groups except male and female Hispanics and Black males. That is, White males and females and Black females experienced a steeper increase in the number of cigarettes smoked per day during early development ( $\beta=0.68$ for White males, $\beta=0.67$ for White females, and $\beta=0.59$ for Black females) and a deceleration in this increase as individuals approached adulthood ( $\beta=-0.74$ for White males; $\beta=-0.63$ for White females; $\beta=-0.61$ for Black females). Among Hispanic females, an increase in ADHD severity was associated with greater number of cigarettes smoked at the intercept, with a relatively less steep slope $(\beta=-0.81, \mathrm{p}=0.001)$ and less deceleration in this slope $(\beta=0.80, \mathrm{p}=0.001)$. Hispanic males showed a similar pattern to their female counterparts, although the slope and quadratic parameters were not significant. For Black males, ADHD severity was not related to the level of cigarettes smoked over age.

The effect of ADHD directionality (HI symptoms in relation to IN symptoms) was related to the count trajectory among White males, Hispanic males and Black females. Specifically, among White males, higher HI symptoms relative to IN symptoms was associated with a significant increase in number of cigarettes smoked over time with a corresponding decrease as individuals approached adulthood $(\beta=0.88, p=.002 ; \beta=-0.90, p<0.001)$. Among Hispanic males, ADHD directionality predicted the number of cigarettes smoked at the intercept $(\beta=0.84, p<0.01)$, the slower linear increase $(\beta=-0.97, p=0.009)$, and less 
deceleration in this increase $(\beta=0.99, p=0.02)$ over developmental age. ADHD directionality was not linked to the count trajectory among Black males and White and Hispanic females.

\subsection{Multi-group Testing of Group (Race and Gender) Comparisons}

Although ADHD severity and directionality predicted the binary and count growth components differently across racial and gender groups, most of these differences were not statistically significantly different between groups. The influences of ADHD symptom severity and directionality on smoking trajectories did not vary significantly between White males and Black males or between Hispanic males and Black males. There were some exceptions. Compared to White males, ADHD severity and directionality among Hispanic males were related to smoking a significantly higher number of cigarettes at the intercept (age 13; $\chi^{2}=5.00, p=0.025 ; \chi^{2}=5.26, p=0.022$ ). Furthermore, ADHD directionality was associated with a faster increase in the number of cigarettes among White males $\left(\chi^{2}=3.38\right.$, $p=0.068)$ compared to Hispanic males.

Regarding the racial differences among females, significant differences were found between Hispanics and Blacks and marginal differences were identified between Hispanics and Whites. In the comparisons between Hispanic and Black females, the differences manifested for the influence of ADHD severity on the intercept, slope, and quadratic for the number of cigarettes smoked $\left(\chi^{2}=4.75 ; p=0.029 ; \chi^{2}=5.75, p=0.017 ; \chi^{2}=5.62, p=0.018\right)$.

Specifically, ADHD severity was correlated to a greater number of cigarettes smoked among Hispanic than Black females at age 13 (intercept). ADHD severity was associated with a greater increase (slope) and deceleration (quadratic) of this increase in the number of cigarettes smoked among Black females compared to Hispanic females. A similar pattern was identified when comparing White females to Hispanic females. That is, ADHD severity was particularly predictive of number of cigarettes at the intercept for Hispanic females compared to White females (age $13 ; \chi^{2}=3.14, p=.076$ ). ADHD severity, however, was associated with faster increase and deceleration of this increase in number of cigarettes smoked among White females than Hispanic females $\left(\chi^{2}=3.16, p=.075 ; \chi^{2}=3.19, p=\right.$. 074). We found no significant differences between White females and Black females.

As for gender differences in smoking within the same racial group, gender differences manifested only within the White group. ADHD severity was linked to a higher likelihood for White males to smoke at a young age (age 13) than White females $\left(\chi^{2}=5.63, p=0.018\right.$ ). However, ADHD severity was associated with the increasing probability of White females smoking during adolescence and emerging adulthood than White males $\left(\chi^{2}=6.16, p=\right.$ 0.013). We found no gender differences in smoking trajectories among Hispanic or Black participants in regards to ADHD symptoms.

\section{DISCUSSION}

To our knowledge, this is the first study to examine the effects of ADHD symptoms on smoking trajectories as functions of race and gender in a U.S. population-based sample. Another unique feature of this study is the simultaneous modeling of both binary and count smoking trajectories. 
The observed cigarette trajectories as a function of race and gender, but independent of ADHD symptoms, are comparable to Chen and Jacobson's (2012) results, which were also derived from Add Health data. Overall, in the present study, a greater proportion of White females had engaged in current smoking at the intercept (age 13) compared to other groups. However, over development, the proportion of White males who smoked exceeded others. Among participants who smoked, White males had the greatest increase in the number of cigarettes smoked across development followed by White females. It is noteworthy that while most racial and gender groups tended to have increased risk of engaging in current smoking during adolescence, the increasing risk of smoking for Black females peaks later, during young adulthood. Also consistent with prior studies (Choi et al., 1997; Flint et al., 1998; Kivinieme et al., 2011), our findings suggest that the risk of current smoking and the number of cigarettes used among Black participants continue into adulthood. These findings emphasize the need to consider race and gender when examining factors predicting smoking trajectories over development.

\subsection{ADHD Severity}

The findings regarding ADHD severity echo previous studies that ADHD symptom severity is an important risk factor for cigarette smoking. The analyses in this study advance this line of work by evaluating severity of ADHD symptoms independent of symptom domain (i.e., HI vs. IN). These data show that greater severity is associated with a higher prevalence of current smoking at age 13 and also with a greater acceleration of cigarette consumption throughout development. Our findings confirm research showing that ADHD symptoms are an independent risk factor for current smoking after controlling for conduct problems (Wilens et al., 2011), and further highlight that ADHD symptom severity, regardless of symptom domain, drives current smoking from age 13-32 in a population-based representative sample in the U.S.

Our findings also point to possible gender and racial differences in the relation between ADHD severity and cigarette smoking. For example, the effect of ADHD severity on the likelihood of smoking at age 13 was particularly robust for White males but less for Black and Hispanic males. Also, ADHD severity was predictive of the increasing number of cigarettes that White males smoked. Moreover, ADHD severity had a stronger influence on the initial number of cigarettes smoked at age 13 among Hispanic males than White males. Similarly, ADHD severity was particularly predictive of the initial number of cigarettes that Hispanic females smoked than White females whereas ADHD severity had a stronger influence on the increasing number of cigarettes for White and Black females than Hispanic females.

These results are consistent with other studies that show that psychological factors differentially related to smoking outcomes as a function of race (Kivinieme et al., 2011; Payne, et al., 2013; Wilson et al., 2014). Thus, continued work is needed to further replicate and extend these findings by examining ADHD severity as a function of these moderating variables. 


\subsection{ADHD Directionality}

Another aim of this study was to examine the influence of HI symptom domain relative to IN. Our findings suggest that after controlling for overall ADHD severity, the HI domain is of greater importance to the initial use and changes in the prevalence of use over development. This might be that impulsive behavior contributes more to current smoking than IN symptoms; whereas the severity of symptoms (inclusive of both IN and HI symptoms) contribute to greater increases in cigarette consumption over development. While other research has also reported that HI symptoms are a better predictor of smoking outcomes than IN symptoms (Chang et al., 2012; Roberts et al., 2014), caution in interpretation is warranted. This is because the mixed findings across previous research regarding relative magnitude of $\mathrm{HI}$ and IN could largely result from several methodological differences such as longitudinal vs. cross-sectional studies, years of observation, and measures of ADHD symptoms and smoking behaviors.

The effects of the IN and HI symptom domains need to be interpreted within the context of gender and race. In our study, ADHD symptom directionality (i.e., having more HI symptoms than IN symptoms) could be particularly predictive for number of cigarettes smoked among Hispanic males at age 13 but not as predictive for young White and Black males. With respect to the risk of engaging in current smoking, our results showed that ADHD directionality was related to an increasing risk of current smoking among Hispanic and Black males but not White males. Moreover, for predicting female smoking behaviors, ADHD directionality was particular predictive for Blacks compared with their counterparts.

\subsection{Implications}

It is not completely clear what contributes to the race and gender disparities in cigarette smoking. As others have hypothesized, the moderating effect of race or gender in the relation between psychological symptoms and smoking could reflect differences in nicotine metabolism across groups (Kivinieme et al., 2011; Lam et al., 2008). These differences might also reflect differential socialization across racial or gender groups. Special effort is needed to assess both biological and socialization differences across race and gender to better understand factors driving the moderating effects of race and gender. It is worth noting that ADHD symptoms in general are not as predictive of smoking behaviors among Black males as others. This might imply that intervention programs for Black males focused on reducing ADHD symptoms as a mean of preventing smoking may not be as fruitful as with other groups.

\subsection{Limitations and Strengths}

Our study should be interpreted in the context of its limitations. First, retrospective ADHD symptoms were limited to recall bias (Mannuzza et al., 2002) and there is a lack of information on ADHD symptoms beyond Wave III when participants reached adulthood. Second, the Add Health study did not have reports from other sources (e.g., teacher, parents) to corroborate the level of ADHD symptoms. However, in previous studies, we have shown that the use of self-report of ADHD symptoms converges with maternal report of the child's history of ADHD diagnosis and medication use (Kollins et al., 2005; McClernon et al., 2008). 
Despite these limitations, our study has important strengths. Our study used a large, nationally representative sample to better understand the relationship between ADHD symptoms and the development of cigarette smoking. The use of population-based samples is needed to replicate findings from clinical samples, which tend to have greater comorbidities and an overrepresentation by White and male participants (Goodman et al, 1997). Future studies are needed to explain the relationship between ADHD symptoms and current smoking as moderated by race and gender.

\section{Acknowledgments}

Role of funding source: This research uses data from Add Health, a program project directed by Kathleen Mullan Harris and designed by J. Richard Udry, Peter S. Bearman, and Kathleen Mullan Harris at the University of North Carolina at Chapel Hill, and funded by grant P01-HD31921 from the Eunice Kennedy Shriver National Institute of Child Health and Human Development, with cooperative funding from 23 other federal agencies and foundations. Special acknowledgment is due Ronald R. Rindfuss and Barbara Entwisle for assistance in the original design. Information on how to obtain the Add Health data files is available on the Add Health website (http:// www.cpc.unc.edu/addhealth). No direct support was received from grant P01-HD31921 for this analysis.

Support to complete analyses and writing this manuscript was received from the following NIH grants: R01 DA030487 awarded to BFF, R01 DA030487 S awarded to BFF to support TC, and K24DA023464 awarded to SHK. The contents of this research are solely the responsibility of the authors and do not represent the official views of NIH or NIDA.

\section{References}

Aytaclar S, Tarter RE, Kirisci L, Lu S. Association between hyperactivity and executive cognitive function in childhood and substance use in early adolescence. J Am Acad Child Adolesc Psychiatry. 1999; 38:172-178. [PubMed: 9951216]

Armstrong TD, Costello EJ. Community studies on adolescent substance use, abuse, or dependence and psychiatric comorbidity. J Consult Clin Psychol. 2002; 70:1224-1239. [PubMed: 12472299]

Bollen, KA.; Curran, PJ. Latent Curve Models: A Structural Equation Perspective. Wiley-Interscience; Hoboken, NJ: 2006.

Burke JD, Loeber R, Lahey BB. Which aspects of ADHD are associated with tobacco use in early adolescence. J Child Psychol Psychiatry. 2001; 42:493-502.10.1111/1469-7610.00743 [PubMed: 11383965]

Chang Z, Lichtenstein P, Larsson H. The effects of childhood ADHD symptoms on early-onset substance use: a Swedish twin study. J Abnorm Child Psychol. 2012; 40:425-435.10.1007/ s10802-011-9575-6 [PubMed: 21947618]

Chen P, Jacobson KC. Developmental trajectories of substance use from early adolescence to young adulthood: gender and racial/ethnic differences. J Adolesc Health. 2012; 50:154-163.10.1016/ j.jadohealth.2011.05.013 [PubMed: 22265111]

Choi WS, Pierce JP, Gilpin EA, Farkas AJ, Berry CC. Which adolescent experimenters progress to established smoking in the United States. Am J Prev Med. 1997; 13:385-391. [PubMed: 9315272]

Duncan SC, Duncan TE, Strycker LA. Alcohol use from ages 9 to 16: a cohort-sequential latent growth model. Drug Alcohol Depend. 2006; 81:71-81. [PubMed: 16006054]

Duncan SC, Duncan TE, Strycker LA, Chaumeton NR. A cohort-sequential latent growth model of physical activity from ages 12 to 17 years. Ann Behav Med. 2007; 33:80-89. [PubMed: 17291173]

Eiraldi RB, Power TJ, Nezu CM. Patterns of comorbidity associated with subtypes of attention-deficit/ hyperactivity disorder among 6- to 12-year-old children. J Am Acad Child Adolesc Psychiatry. 1997; 36:503-514. [PubMed: 9100425]

Essex MJ, Kraemer HC, Armstrong JM, Boyce WT, Goldsmith HH, Klein MH, Woodward H, Kupfer DJ. Exploring risk factors for the emergence of children's mental health problems. Arch Gen Psychiatry. 2006; 63:1246-1256. [PubMed: 17088505] 
Flint AJ, Yamada EG, Novotny TE. Black-White differences in cigarette smoking uptake: progression from adolescent experimentation to regular use. Prev Med. 1998; 27:358-364.10.1006/pmed. 1998.0299 [PubMed: 9612826]

Fuemmeler BF, Kollins SH, McClernon FJ. Attention deficit hyperactivity disorder symptoms predict nicotine dependence and progression to regular smoking from adolescence to young adulthood. $\mathrm{J}$ Pediatr Psychol. 2007; 32:1203-1213.10.1093/jpepsy/jsm051 [PubMed: 17602186]

Fuemmeler BF, Lee CT, Ranby KW, Clark T, McClernon FJ, Yang C, Kollins SH. Individual- and community-level correlates of cigarette-smoking trajectories from age 13 to 32 in a U.S. population-based sample. Drug Alcohol Depend. 2013; 132:301-308.10.1016/j.drugalcdep. 2013.02.021 [PubMed: 23499056]

Goodman S, Lahey B, Fielding B, Dulcan M, Narrow W, Regier D. Representativeness of clinical samples of youths with mental disorders: a preliminary population-based study. J Abnorm Psychol. 1997; 106:3-14.10.1037//0021-843X.106.1.3 [PubMed: 9103713]

Gudjonsson GH, Sigurdsson JF, Sigfusdottir ID, Young S. An epidemiological study of ADHD symptoms among young persons and the relationship with cigarette smoking, alcohol consumption and illicit drug use. J Child Psychol Psychiatry. 2012; 53:304-312.10.1111/j. 1469-7610.2011.02489.x [PubMed: 22066497]

Harris, K.; Halpern, C.; Whitsel, E.; Hussey, J.; Tabor, J.; Entzel, P.; Udry, J. The National Longitudinal Study Of Adolescent Health: Research Design. North Carolina Population Center; Chapel Hill, NC: 2009.

Kandel D, Schaffran C, Hu MC, Thomas Y. Age-related differneces in cigarette smoking among Whites and African-Americans: evidence for the crossover hypothesis. Drug Alcohol Depend. 2011; 118:280-287. [PubMed: 21561724]

Kivinieme MT, Orom H, Giovino GA. Psychological distress and smoking behavior: the nature of the relation differs by race/ethnicity. Nicotine Tob Res. 2011; 13:113-119. [PubMed: 21159784]

Kollins SH, McClernon FJ, Fuemmeler BF. Association between smoking and attention-deficit/ hyperactivity disorder symptoms in a population-based sample of young adults. Arch Gen Psychiatry. 2005; 62:1142-1147.10.1001/archpsyc.62.10.1142 [PubMed: 16203959]

Lam CY, Robinson JD, Carter BL, Wetter DW, Minnix JA, Cinciripini PM. Nicotine differentially inhibits the acoustic startle reflex in African American and Caucasian American smokers. Addict Behav. 2008; 33:1521-1528.10.1016/j.addbeh.2008.07.006 [PubMed: 18771859]

Mannuzza S, Klein RG, Klein DF, Bessler A, Shrout P. Accuracy of adult recall of childhood attention deficit hyperactivity disorder. Am J Psychiatry. 2002; 159:1882-1888.10.1176/appi.ajp. 159.11.1882 [PubMed: 12411223]

McClernon FJ, Fuemmeler BF, Kollins SH, Kail ME, Ashley-Koch AE. Interactions between genotype and retrospective ADHD symptoms predict lifetime smoking risk in a sample of young adults. Nicotine Tob Res. 2008; 10:117-127.10.1080/14622200701704913 [PubMed: 18188752]

Miles DR, van den Bree M, Pickens RW. Sex differences in shared genetic and environmental influences between conduct disorder symptoms and marijuana use in adolescents. Am J Med Genet. 2002; 114:159-168. [PubMed: 11857577]

Molina BSG, Pelham WE Jr. Childhood pedictors of adolescent substauce use in a logitudinal study of children with ADHD. J Abnorm Psychol. 2003; 112:497-507. [PubMed: 12943028]

Muthén, LK.; Muthén, BO. Mplus User's Guide. 7. Los Angeles, CA: 1998-2012.

Payne TJ, Ma JZ, Crews KM, Li MD. Depressive symptoms among heavy cigarette smokingers: the influence of daily rate, gender, and race. Nicotine Tob Res. 2013; 15:1714-1721. [PubMed: 23569006]

Polanczyk G, Caspi A, Houts R, Kollins SH, Rohde LA, Moffitt TE. Implications of extending the ADHD age-of-onset criterion to age 12: results from a prospectively studied birth cohort. J Am Acad Child Adolesc Psychiatry. 2010; 49:210-216. [PubMed: 20410710]

Ranby KW, Boynton MH, Kollins SH, McClernon FJ, Yang C, Fuemmeler BF. Understanding the phenotypic structure of adult retrospective ADHD symptoms during childhood in the United States. J Clin Child Psychol. 2012; 41:261-274.10.1080/15374416.2012.654465

Resnick MD, Bearman PS, Blum RW, Bauman KE, Harris KM, Jones J, Tabor J, Beuhring T, Sieving RE, Shew M, Ireland M, Bearinger LH, Udry JR. Protecting adolescents from harm: findings from 
the national longitudinal study on adolescent health. JAMA. 1997; 278:823-832. [PubMed: 9293990]

Roberts W, Peters JR, Adams ZW, Lynam DR, Millich R. Identifying the facets of impulsivity that explain the relation between ADHD symptoms and substance use in a nonclinical sample. Addict Behav. 2014; 39:1272-1277. [PubMed: 24813555]

Substance Abuse and Mental Health Services Administration. Results from the 2011 National Survey on Drug Use and Health: Summary of National Findings. Rockville, MD: 2012. NSDUH Series H-44, HHS Publication No. (SMA) 12-4713

Tercyak KP, Lerman C, Audrain J. Association of attention-deficit/hyperativivity disorder symptoms with levels of cigarette smoking in a community sample of adolescents. J Am Acad Child Adolesc Psychiatry. 2002; 41:799-805. [PubMed: 12108804]

Van Voorhees EE, Mitchell JT, McClernon FJ, Beckham JC, Kollins SH. Sex, ADHD symptoms, and smoking outcomes: an integrative model. Med Hypotheses. 2012; 78:585-593.10.1016/j.mehy. 2012.01.034 [PubMed: 22341778]

White HR, Nagin D, Replogle E, Stouthamer-Loeber M. Racial differences in trajectories of cigarette use. Drug Alcohol Depend. 2004; 76:219-227. [PubMed: 15561473]

Willoughby MT, Kollins SH, McClernon FJ. Association between smoking and retrospectively reported attention-deficit/hyperactivity disorder symptoms in a large sample of new mothers. Nicotine Tob Res. 2009; 11:313-322.10.1093/ntr/ntp001 [PubMed: 19307443]

Wilson SM, Dedert EA, Dennis PA, Dennis MF, Calhoun PS, Kirby AC, Beckham JC. Do ethnicity and gender moderate the influence of posttraumatic stress disoer on time to smoking lapse. Addict Behav. 2014; 39:1163-1167. [PubMed: 24727113] 


\section{Highlights}

- Attention Deficit Hyperactivity Disorder (ADHD) severity and directionality had unique effects on smoking trajectories.

- ADHD severity is associated with prevalence of smoking at a young age (age 13).

- ADHD severity is related to the acceleration of cigarette consumption.

- ADHD directionality is related to the increasing prevalence of smoking.

- The risk of ADHD symptoms may differ by race and gender. 


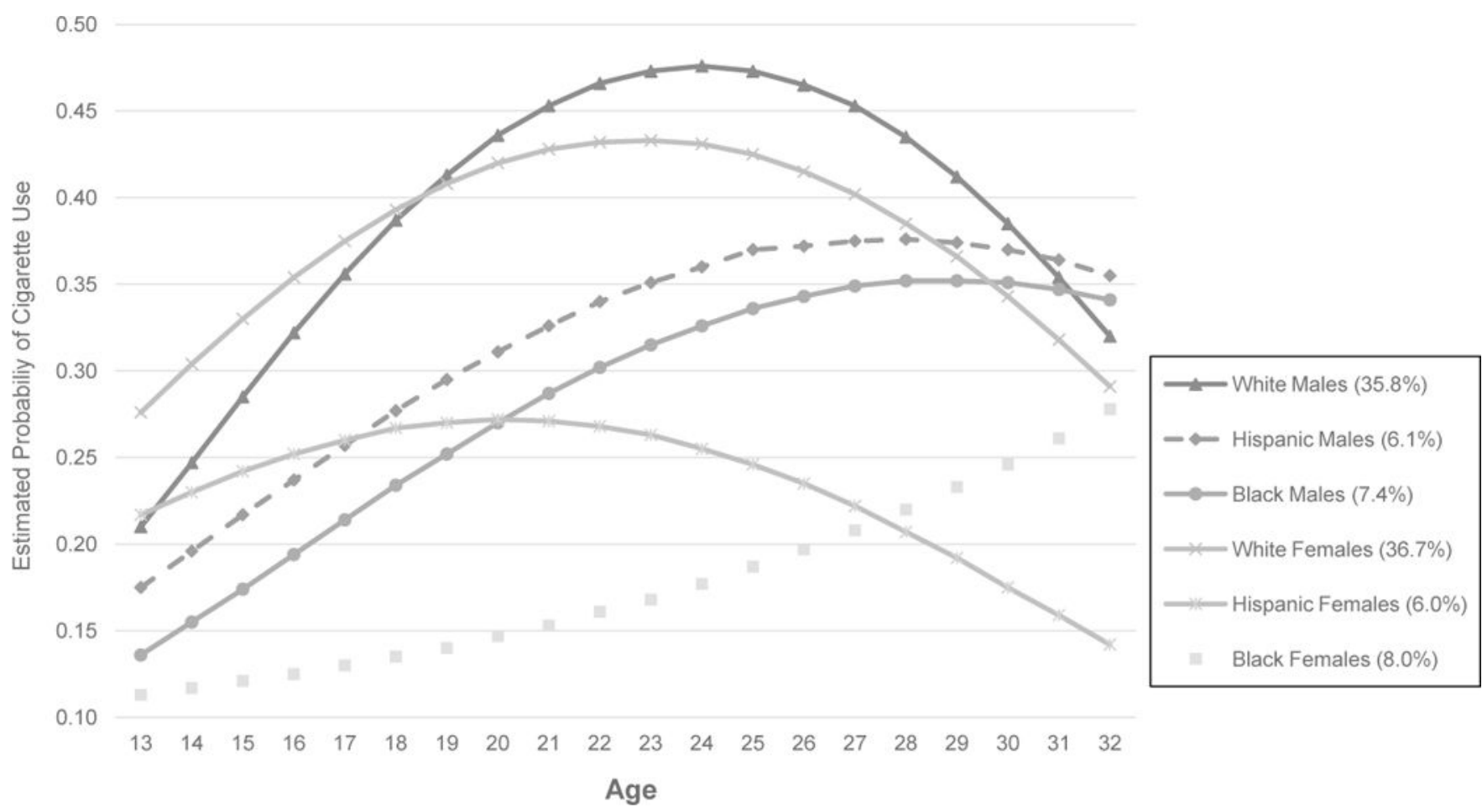

Fig. 1.

Estimated trajectory for the probability of current smoking stratified by race and gender $(\mathrm{N}$ $=9,719)$. 


$$
8.00
$$

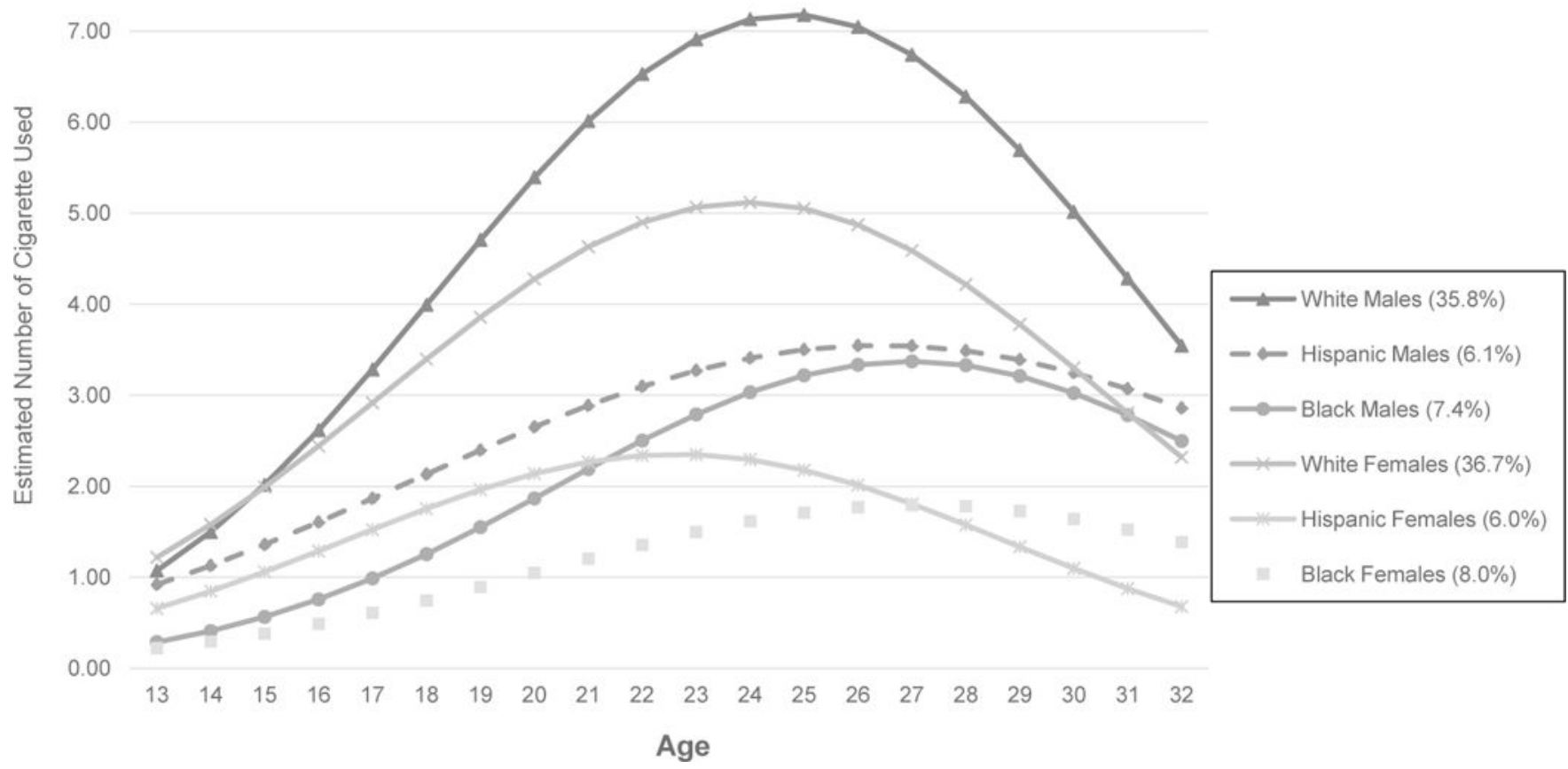

Fig.2.

Estimated trajectory for the number of cigarettes used stratified by race and gender $(\mathrm{N}=$ 9,719).

Drug Alcohol Depend. Author manuscript; available in PMC 2016 March 01. 


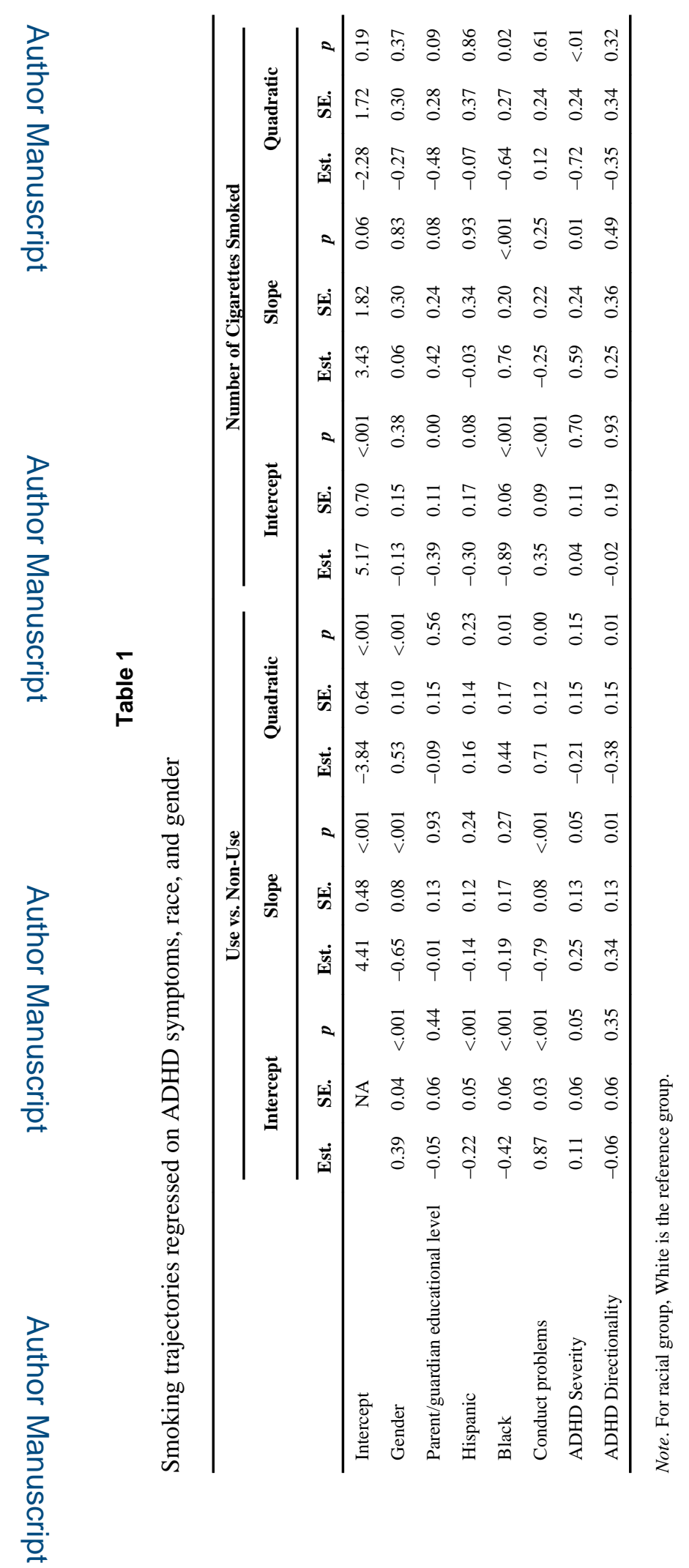

Drug Alcohol Depend. Author manuscript; available in PMC 2016 March 01. 


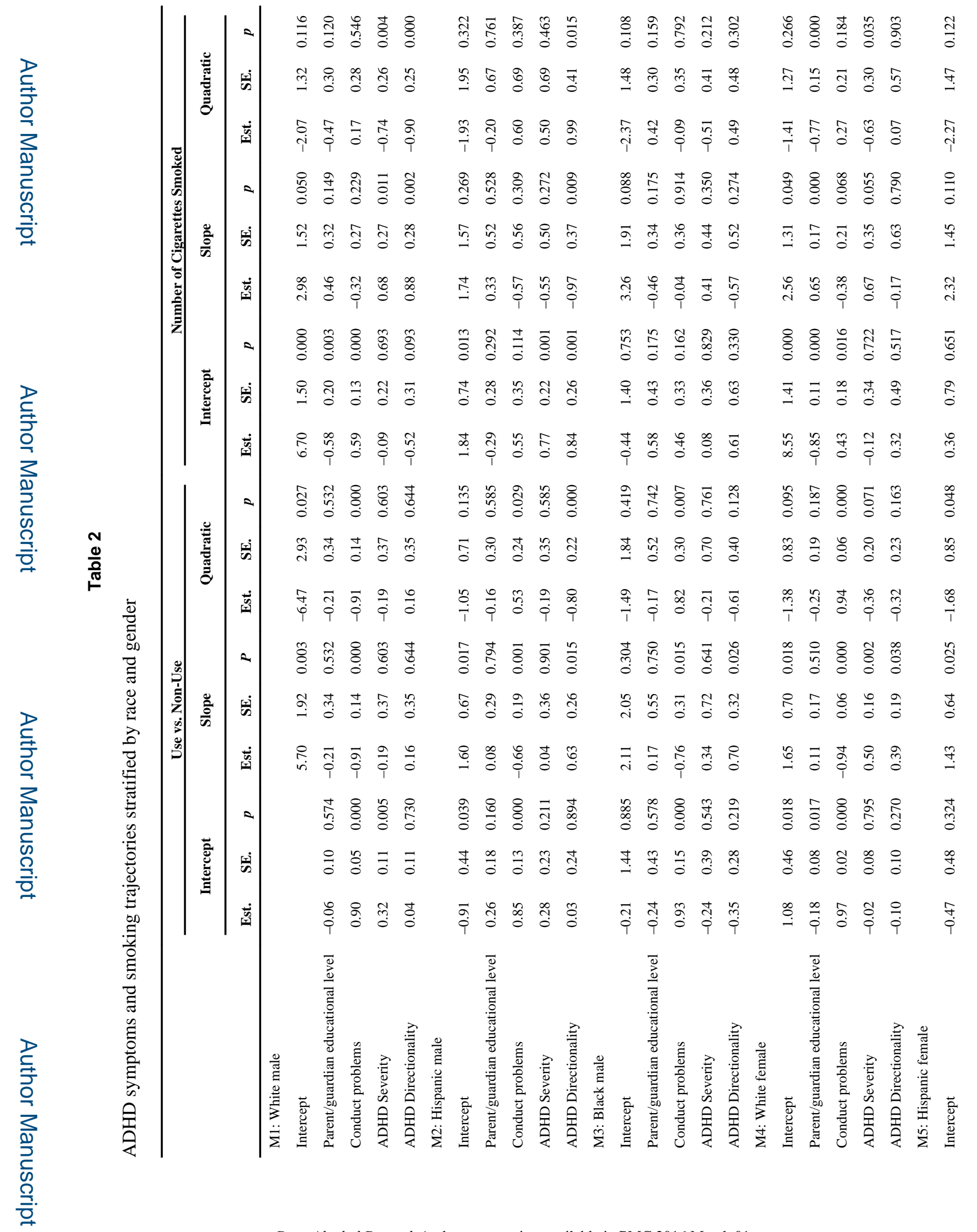

Drug Alcohol Depend. Author manuscript; available in PMC 2016 March 01. 


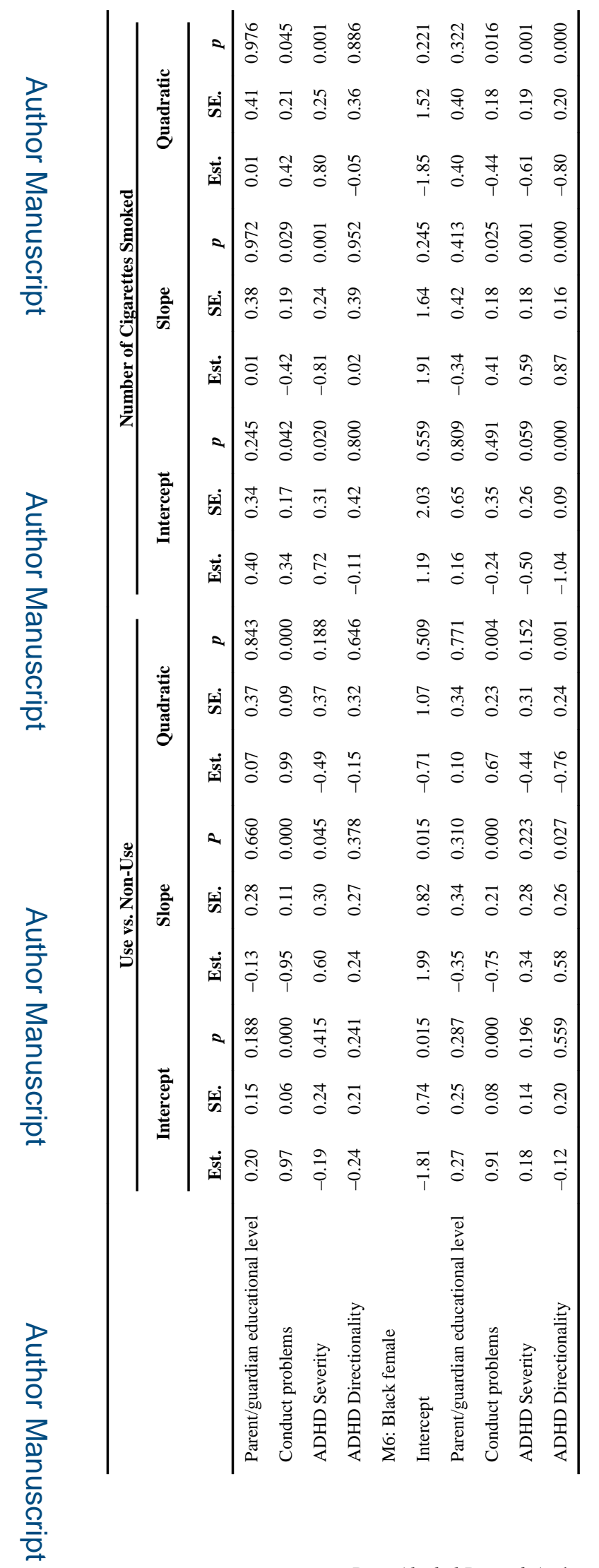

Drug Alcohol Depend. Author manuscript; available in PMC 2016 March 01. 